\title{
Systematic search for putative new domain families in Mycoplasma gallisepticum genome
}

\author{
Chilamakuri CS Reddy ${ }^{1,2}$, Sane Sudha Rani ${ }^{2}$, Bernard Offmann ${ }^{1 *}$, R Sowdhamini ${ }^{2^{*}}$
}

\begin{abstract}
Background: Protein domains are the fundamental units of protein structure, function and evolution. The delineation of different domains in proteins is important for classification, understanding of structure, function and evolution. The delineation of protein domains within a polypeptide chain, namely at the genome scale, can be achieved in several ways but may remain problematic in many instances. Difficulties in identifying the domain content of a given sequence arise when the query sequence has no homologues with experimentally determined structure and searching against sequence domain databases also results in insignificant matches. Identification of domains under low sequence identity conditions and lack of structural homologues acquire a crucial importance especially at the genomic scale.
\end{abstract}

Findings: We have developed a new method for the identification of domains in unassigned regions through indirect connections and scaled up its application to the analysis of 434 unassigned regions in 726 protein sequences of Mycoplasma gallisepticum genome. We could establish 71 new domain relationships and probable 63 putative new domain families through intermediate sequences in the unassigned regions, which importantly represent an overall 10\% increase in PfamA domain annotation over the direct assignment in this genome.

Conclusions: The systematic analysis of the unassigned regions in the Mycoplasma gallisepticum genome has provided some insight into the possible new domain relationships and putative new domain families. Further investigation of these predicted new domains may prove beneficial in improving the existing domain prediction algorithms.

\section{Background}

Domain assignment to the protein sequences has paramount importance in the post genomic era. Protein domains are the structural, functional and evolutionary units of proteins. Study of proteins at the domain level has had a profound impact on the study of individual proteins. Experimental and/or computational methods can be used to identify domains in the given protein sequence. Classification databases such as the Dali Domain Dictionary[1], CATH[2], SCOP[3] and DIAL[4] employ structural data to locate and assign domains. Identification of domains at the sequence level depends on the detection of global and local sequence similarities between a given query sequence and domain sequences

\footnotetext{
*Correspondence: bernard.offmann@univ-reunion.fr; mini@ncbs.res.in 1Université de La Réunion, Equipe de Bioinformatique, Laboratoire de
Biochimie et Génétique Moléculaire, 15 ave René Cassin, 97715 Saint Den Université de La Réunion, Equipe de Bioinformatique, Laboratoire de
Biochimie et Génétique Moléculaire, 15 ave René Cassin, 97715 Saint Denis Messag Cedex 09, La Réunion, France

${ }^{2}$ National Centre for Biological Sciences, GKVK Campus, Bellary Road, 560065 Bangalore, India
}

(C) 2010 Offmann et al; licensee BioMed Central Ltd. This is an Open Access article distributed under the terms of the Creative Commons Attribution License (http://creativecommons.org/licenses/by/2.0), which permits unrestricted use, distribution, and reproduction in any medium, provided the original work is properly cited. tionary divergence, it is not always possible to identify distantly related protein domains by sequence search techniques. The realization of additional domains in those circumstances can be tedious, involving manual intervention, but can lead to better understanding of overall biological function. We have recently introduced an automatic multi-step approach, PURE for recognizing such connections[6,7].

Mycoplasma gallisepticum causes chronic respiratory disease in chickens and other avian species. The infections result in considerable economic losses in poultry production. This pathogen has a small genome with 726 proteins [8], but only 498 protein sequences have known Pfam hits with $46 \%$ residue coverage [9]. The gap in the annotation of this genome emphasizes the need for further exploration for other methods for domain assignment from sequence. We have recently shown that it is possible to enhance prediction of 
domains in the unassigned regions by $25 \%$ through indirect connections in the class III adenylyl cyclase domain containing proteins [10]. Here, we demonstrate that this method can be scaled up for whole genome analysis, by taking Mycoplasma gallisepticum genome as a specific example.

\section{Results and Discussion}

The procedure that was followed in this study and overall results are described in Figure 1. Initially, there are 726 proteins in the Mycoplasma gallisepticum genome; domains were assigned to the genome with HMMpfam program of HMMER package [11] by search against Pfam 21 databases [5,12] with expectation value cut off of 0.01 . Only PfamA families are used in HMMpfam search, since PfamB families were generated automatically and have no associated annotation, references and relatively low quality data than PfamA families [12]. The regions in protein sequences, which are associated with domains, are called 'assigned regions'; rest of the regions without PFAM domain assignment are called 'unassigned regions'. There were 620 unassigned regions in the 726 proteins. To avoid false positives in the similarity search, the unassigned regions with at least 70 residues long were considered. In our analysis, 434 unassigned regions have at least 70 residues long. Transmembrane, coiled-coils and low complexity regions were excluded and only 364 sequences passed through this filtering step. A further filter was placed to ensure adequate secondary structural content giving rise to 359 sequences. $15 \%$ cut-off on predicted structural content was chosen as the minimum value, consistent with our earlier work [13]. Only 230 out of 359 unassigned regions picked up at least two hits in PSI-BLAST search; full-length sequences for all the non-self hits were obtained and used as query for HMMpfam search to assign domains to the sequences. Only 62 of 230 unassigned regions were associated with PfamA domains through homologous sequences and remaining 168 unassigned sequences failed to pick up domain associations. These 62 unassigned sequences correspond to 71 newly

\section{Methodology}

Total 726 protein sequences with 620 unassigned regions, but only- 434
have a length $>=70$ residues

Filtered for Transmembrane, coiled-coil regions- 364

Secondary Structure Prediction (PSIPRED)- 359

PSI-BLAST search against NR database with E value 0.001

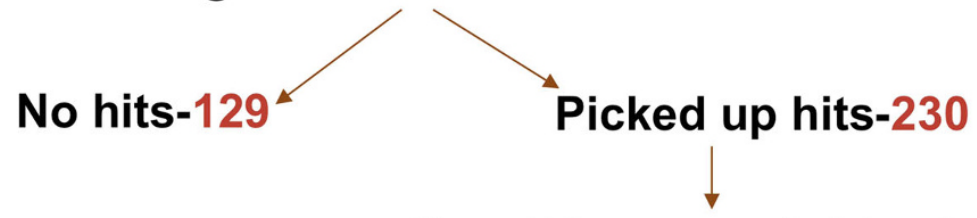

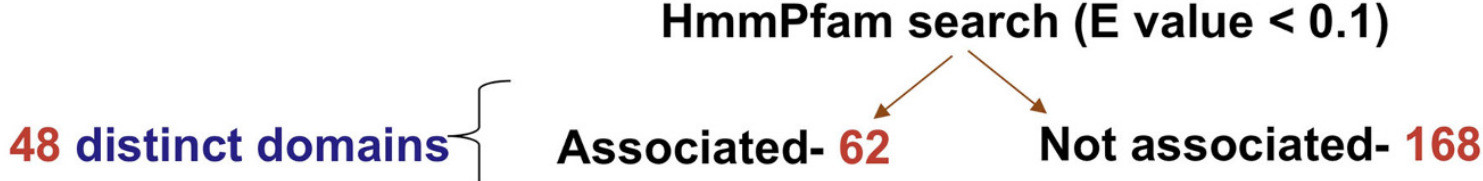

Figure 1 Methodology of the approach and statistics. Initially, 726 protein sequences were considered from Mycoplasma gallisepticum genome, which have 620 unassigned regions of different lengths. 434 unassigned regions are at least 70 residues long. Out of 434 , only 364 passed through transmembrane and coiled coil filtering and 359 sequences after secondary structure filtering. The remaining unassigned regions (359) sequences were subject to PSI-BLAST searches, but only 230 unassigned regions picked up at least two hits. We extracted full-length sequences for each hit in PSI-BLAST and used for HMMpfam search. Here again, only 62 unassigned regions were associated indirectly with preexisting domains which correspond to 48 different domain families. 
Table 1 Newly predicted domains in the Mycoplasma gallisepticum genome

\begin{tabular}{lll}
\hline S. & Domain Name & Description \\
\hline 1. & AAA & ATPase family associated with various cellular activities \\
\hline 2. & Anticodon_1 & $\begin{array}{l}\text { Anticodon-binding domain. This domain is found valyl and leucyl } \\
\text { tRNA synthetases. It binds to the anticodon of the tRNA. }\end{array}$
\end{tabular}

\section{Full}

NP_853502.1 = 9-182

NP_852939.1 $=397-590$

NP_852935.1 $=55-218$

NP_853215.1 $=620-850$

\begin{tabular}{|c|c|c|c|c|}
\hline 3. & ATP-synt_ab_C & ATP synthase alpha/beta chain, $C$ terminal domain. & NP_853478.1 = 140-221 & - \\
\hline 4. & ATP-synt_ab_N & ATP synthase alpha/beta family, beta-barrel domain & $\begin{array}{l}\text { NP_853438.1 }=4-126 \\
\text { NP_853439.1 }=2-125\end{array}$ & - \\
\hline 5. & BPD_transp_1 & $\begin{array}{l}\text { Binding-protein-dependent transport system inner membrane } \\
\text { component. }\end{array}$ & $\begin{array}{l}\text { NP_853029.1 }=53-260 \\
\text { NP_853249.1 }=59-232\end{array}$ & - \\
\hline 6. & CHASE-3 \# & $\begin{array}{l}\text { Cyclases/Histidine kinases Associated Sensory Extracellular) present } \\
\text { in diverse receptor-like proteins with histidine kinase and } \\
\text { nucleotide cyclase domains }\end{array}$ & NP_853387.1 = 55-582 & - \\
\hline 7. & DUF-30 & Domain of Unknown Function 30 & NP_853479.1 = 370-770 & - \\
\hline 8. & DUF-31 & Domain of Unknown Function 31 & $\begin{array}{l}\text { NP_853440.1 }=220-317 \\
\text { NP_853441.1 }=233-337 \\
\text { NP_853488.1 }=220-340\end{array}$ & - \\
\hline 9. & LMP \$ & $\begin{array}{l}\text { LMP repeated region. Found in the LMP group of surface-located } \\
\text { membrane proteins of Mycoplasma hominis. }\end{array}$ & $\begin{array}{l}\text { NP_853333.1 = 1260-1320, } \\
1420-1580,1600-1760\end{array}$ & - \\
\hline 10. & Ferritin $\$$ & $\begin{array}{l}\text { Ferritin-like domain is one of the major non-haem iron storage } \\
\text { proteins in animals, plants, and microorganisms }\end{array}$ & NP_852976.1 = 5-143 & - \\
\hline 11. & GMP_synt_C \$ & GMP synthase $C$ terminal domain. & NP_852801.1 = 220-275 & - \\
\hline 12. & Helicase_C & $\begin{array}{l}\text { Helicase conserved C-terminal domain. Found in a wide variety of } \\
\text { helicases and helicase related proteins. }\end{array}$ & $\begin{array}{l}\text { NP_852813.1 }=440-530 \\
\text { NP_853467.1 }=660-730\end{array}$ & - \\
\hline 13. & HGTP_anticodon & $\begin{array}{l}\text { Anticodon binding domain. tRNA synthetases, or tRNA ligases are } \\
\text { involved in protein synthesis. This domain is found in histidyl, } \\
\text { glycyl, threonyl and prolyl tRNA synthetases. }\end{array}$ & NP_852966.1 $=342-423$ & - \\
\hline 14. & $\mathrm{HHH} \#$ & $\begin{array}{l}\text { The helix-hairpin-helix DNA-binding motif is found to be } \\
\text { duplicated in the central domain of RuvA. }\end{array}$ & $\begin{array}{l}\text { NP_853482.1 }=589-619 \\
\text { NP_853386.1 }=63-92,98-127\end{array}$ & - \\
\hline 15. & HTH_11 \# & Helix-turn-helix domain present in a wide variety of proteins. & NP_853136.1 = 28-73 & - \\
\hline$\overline{16 .}$ & HTH_12 \# & $\begin{array}{l}\text { Ribonuclease } R \text { winged-helix domain. Found found at the amino } \\
\text { terminus of Ribonuclease } R \text { and a number of presumed } \\
\text { transcriptional regulatory proteins from archaea. }\end{array}$ & NP_853240.1 = 38-89 & - \\
\hline 17. & S1 & $\begin{array}{l}\text { The S1 domain occurs in a wide range of RNA associated } \\
\text { proteins. It is structurally similar to cold shock protein which binds } \\
\text { nucleic acids. The S1 domain has an OB-fold structure. }\end{array}$ & NP_852895.1 = 140-210 & - \\
\hline 18. & KH_1 & $\begin{array}{l}\text { The } \mathrm{K} \text { homology }(\mathrm{KH}) \text { domain was first identified in the human } \\
\text { heterogeneous nuclear ribonucleoprotein (hnRNP) K. It is a } \\
\text { domain of around } 70 \text { amino acids that is present in a wide variety } \\
\text { of quite diverse nucleic acid-binding proteins. }\end{array}$ & NP_852895.1 = 333-393 & - \\
\hline 19. & Lactamase_B & Metallo-beta-lactamase superfamily. & NP_852865.1 $=40-248$ & - \\
\hline 20. & RMMBL & RNA-metabolising metallo-beta-lactamase. & NP_852865.1 = 320-360 & - \\
\hline 21. & Lipoprotein_17 & Lipoprotein associated domain. & NP_852799.1 $=49-160$ & - \\
\hline 22. & MatE & $\begin{array}{l}\text { Multi Antimicrobial Extrusion (MATE) family function as drug/ } \\
\text { sodium antiporters. }\end{array}$ & NP_853011.1 = 364-530 & - \\
\hline 23. & Methyltransf_3 \$ & O-methyltransferase & NP_852906.1 = 7-185 & - \\
\hline 24. & MFS_1 \$ & Major Facilitator Superfamily & NP_852970.1 = 480-922 & - \\
\hline 25. & NusB \$ & $\begin{array}{l}\text { The NusB protein is involved in the regulation of rRNA } \\
\text { biosynthesis by transcriptional antitermination. }\end{array}$ & NP_853291.1 = 13-130 & - \\
\hline 26. & Peptidase_M23 \$ & Peptidase family M23 & NP_853190.1 = 484-657 & - \\
\hline 27. & PGM_PMM_IV \$ & Phosphoglucomutase/phosphomannomutae, C-terminal domain & NP_853364.1 = 481-550 & - \\
\hline 28. & PTS_EIIB & phosphotransferase system, EIIB & NP_853326.1 = 47-85 & - \\
\hline 29. & SBP_bac_1\$ & Bacterial extracellular solute-binding protein & NP_852821.1 = 1-385 & NP_852814.1 = 6-181 \\
\hline
\end{tabular}

Partial

$-$


Table 1: Newly predicted domains in the Mycoplasma gallisepticum genome (Continued)

\begin{tabular}{|c|c|c|c|c|}
\hline 30. & Sigma70_r1_1 \# & Sigma-70 factor, region 1.1. & NP_853171.1 = 288-342 & - \\
\hline 31. & Sigma70_r1_2\$ & Sigma-70 factor, region 1.2 & NP_853171.1 = 357-398 & - \\
\hline 32. & Sigma70_r4_2\$ & Sigma-70, region 4 & NP_852863.1 = 120-170 & - \\
\hline 33. & TGS \$ & ThrRS, GTPase, and SpoT domain. & NP_852968.1 = 417-487 & - \\
\hline 34. & THUMP \$ & thiouridine synthases, methylases and PSUSs domain. & NP_853282.1 = 78-170 & - \\
\hline 35. & Transketolase_C & $\begin{array}{l}\text { The C-terminal domain of transketolase has been proposed as a } \\
\text { regulatory molecule binding site }\end{array}$ & $\begin{array}{l}\text { NP_852812.1 }=530-641 \\
\text { NP_853134.1 }=138-262\end{array}$ & - \\
\hline 36. & tRNA_anti & OB-fold nucleic acid binding domain & NP_852876.1 = 230-310 & - \\
\hline 37. & VapD_N \# & Virulence-associated protein D & NP_853458.1 = 7-49 & - \\
\hline 38. & Lipoprotein_X & Mycoplasma MG185/MG260 protein. & - & $\begin{array}{l}\text { NP_852988.1 }=247-404 \\
\text { NP_852899.1 }=257-414\end{array}$ \\
\hline 39. & Lipoprotein_10 & Putative mycoplasma lipoprotein, C-terminal region & NP_852988.1 = 444-563 & - \\
\hline 40. & DEAD & $\begin{array}{l}\text { Members of this family include the DEAD and DEAH box } \\
\text { helicases. Helicases are involved in unwinding nucleic acids. }\end{array}$ & - & NP_852877.1 = 596-722 \\
\hline 41. & ABC_membrane & ABC transporter transmembrane region. & - & NP_852786.1 = 2-126 \\
\hline 42. & ABC_tran & ABC transporter & - & NP_853051.1 = 317-467 \\
\hline & DUF258 & Domain of Unknown Function 258 & - & NP_853404.1 = 7-104 \\
\hline & GTP_EFTU & & - & NP_853200.1 = 68-151 \\
\hline 45. & RecO \# & Recombination protein $\mathrm{O}$ & - & NP_853174.1 = 1-74 \\
\hline 46. & SBP_bac_5 \$ & & - & NP_853298.1 = 461-889 \\
\hline 47. & Transposase_mut & Transposase, Mutator family & - & $\begin{array}{l}\text { NP_852891.1 }=6-108 \\
\text { NP_853257.1 }=16-83 \\
\text { NP_852883.1 }=2-121\end{array}$ \\
\hline 48. & $\mathrm{HNH} \$$ & $\mathrm{HNH}$ endonuclease & NP_853456.1 = 650-708 & - \\
\hline
\end{tabular}

Among probable new domains, some of the them are first time identified in Mycoplasma gallisepticum genome (indicated by $\$$ or \#). Among those unique domains, some are not even present in other Mycoplasmas (indicated by \#), while some of them are present in other Mycoplasmas (indicated by \$). Second column indicates the name of the domain, third column a brief description about the domain and fourth and fifth columns indicate the kind of association which may be full association (we refer 'fully associated' when at least $75 \%$ of unassigned region is indirectly aligned with domain region) or partial association (we call partial associated when at most $75 \%$ of unassigned region is indirectly aligned with domain region). Under full and partial columns, we indicate the protein id and start and end residue number of probable new domain.

\# Unique domain, this domain was not presently identified in entire Mycoplasmataceae family.

\$ Unique domain but present in other Mycoplasmataceae family members.

predicted domains out of which 58 were fully associated and 13 were partially associated. In the fully associated domains, at least $75 \%$ of Pfam domain should lie within the unassigned region, otherwise it is called as 'partially associated domain'.

These 71 newly predicted domains belong to 48 unique domain families, but among the newly predicted 48 unique domain families, only 22 unique domains were initially not present in the Mycoplasma gallisepticum genome; for the remaining 26 domains, one or more copies already exist in the genome. Among the new predictions, 22 domains appear specific to M. gallisepticum genome, only 15 domains were present in other Mycoplasmataceae members and remaining 7 domains are unique to the entire Mycoplasmataceae family members (Table 1 ).

To validate the newly predicted domains, we generated multiple sequence alignments using CLUSTALW program [14]. Inputs for multiple sequence alignment are the unassigned sequence and the representative sequences of the newly assigned domain obtained from Pfam database. In most cases, only few family-specific signature residues are conserved, suggesting extreme levels of evolutionary divergence from classical members of such Pfam families.

The number of newly predicted domains was substantial; it raises an interesting question: why were these domains not annotated in the initial search? It is likely because of the poor sequences identities between query and hit. Though sequence analysis-based remote-homology detection approaches, such as Hidden Markov Models (HMMs), are powerful tools, these methods often face limitations due to poor sequence similarities and non-uniform sequence dispersion in protein sequence space. Several interesting approaches have been employed in different ways to detect remotely related proteins; one such approach is based on the intermediate sequences. Intermediate sequence procedure 
substantially increases the ability to recognize the distant evolutionary relationships[15].

There is relatively large number (63) of unassigned regions, which has picked up at least five homologues but not associated with any PfamA domain (Additional file 1: Supplemental Table S1). We examined below few examples of these regions, which can be regarded as potentially putative new domain families. Search against PfamB profile HMM of Pfam24 database showed that 20 unassigned regions, where putative new domain families were predicted by us, were also associated with at least one PfamB domain (Additional file 2: Supplemental Table S2). However, about two-third (43 out of 63 unassigned regions) neither have a hit in the PfamA database nor in the PfamB database. These 43 regions may indicate potential new domain families, which are yet to be annotated in the Pfam database (Additional file 2: Supplemental Table S2).

The NP_853398.1 protein (Figure 2, Figure 3) sequence, which is 329 residues long, has a single asparagine synthetase (AsnA) domain from 5 to 241 residues and one $\mathrm{C}$-terminus unassigned region from 242 to 329 . When the unassigned region of this protein (242-329) was analyzed, based on the intermediate sequences using the methodology described above, 106 similar sequences were identified in the PSI-BLAST search and these hit sequences were from both prokaryotes and eukaryotes. In the HMMpfam search, however, it was not associated with any PfamA domain, rather it was associated with PfamB_3316 domain. This unassigned region has about $62 \%$ predicted secondary structural content with 5 helices and $3 \beta$ strands. More interestingly, the predicted $\beta-3 \alpha-\beta-\alpha-\beta$ $\alpha$ secondary structure pattern is conserved in all the homologous sequences. All the homologous sequences have similar domain architecture. The crystal structure of E.coli asparagine synthetase also showed the presence of this small subdomain[16]. Aspartate-ammonia ligase (asparagine synthetase) catalyses the conversion of L-aspartate to L-asparagine in the presence of ATP and ammonia. Asn A structure revealed that AsnA structure is similar to that of the catalytic domain of yeast aspartyl-tRNA synthetase despite low sequence similarity. These enzymes have a common reaction mechanism that implies the formation of an aminoacyl-adenylate intermediate. The cluster of highly conserved residues (GGGIG) motif plays an important role in the formation of a cavity which can accommodate bound ATP in aspartyl-tRNA synthetase[16]. Since this motif is conserved in the newly predicted putative domain, it may play an important role in the ligand binding.

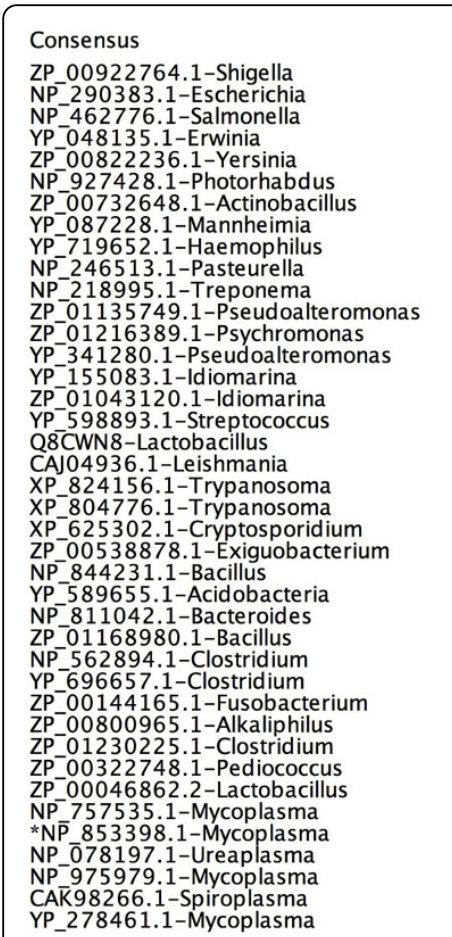

Figure 2 Multiple sequence alignment of unassigned region (NP_853398.1.-242-329) and its homologues obtained in PSI-BLAST search. Unassigned region indicated by ${ }^{\prime * \prime}$ mark and consensus sequence is shown on the top of the alignment. Species name is given along with sequence ids. The highly conserved GGGIG motif is highlighted.

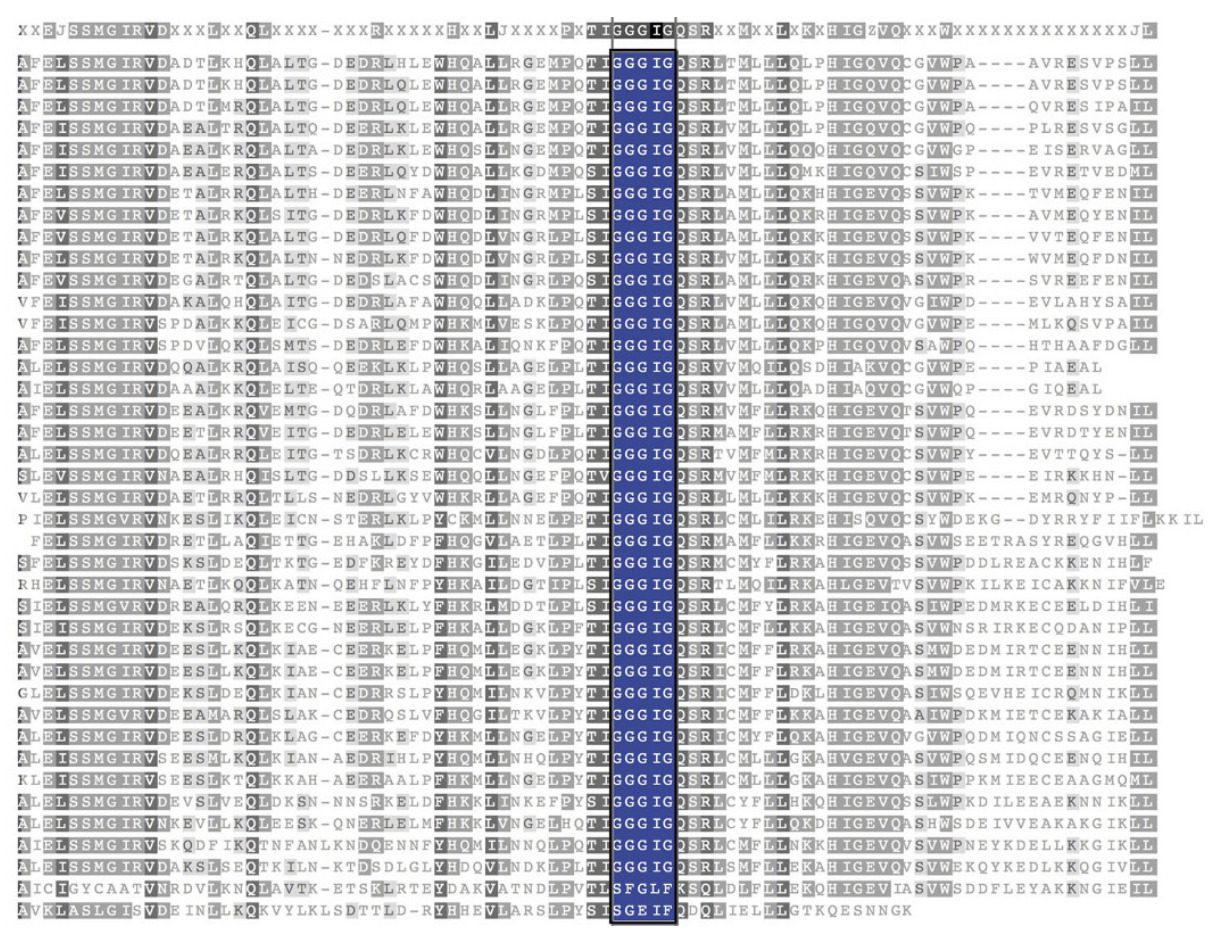




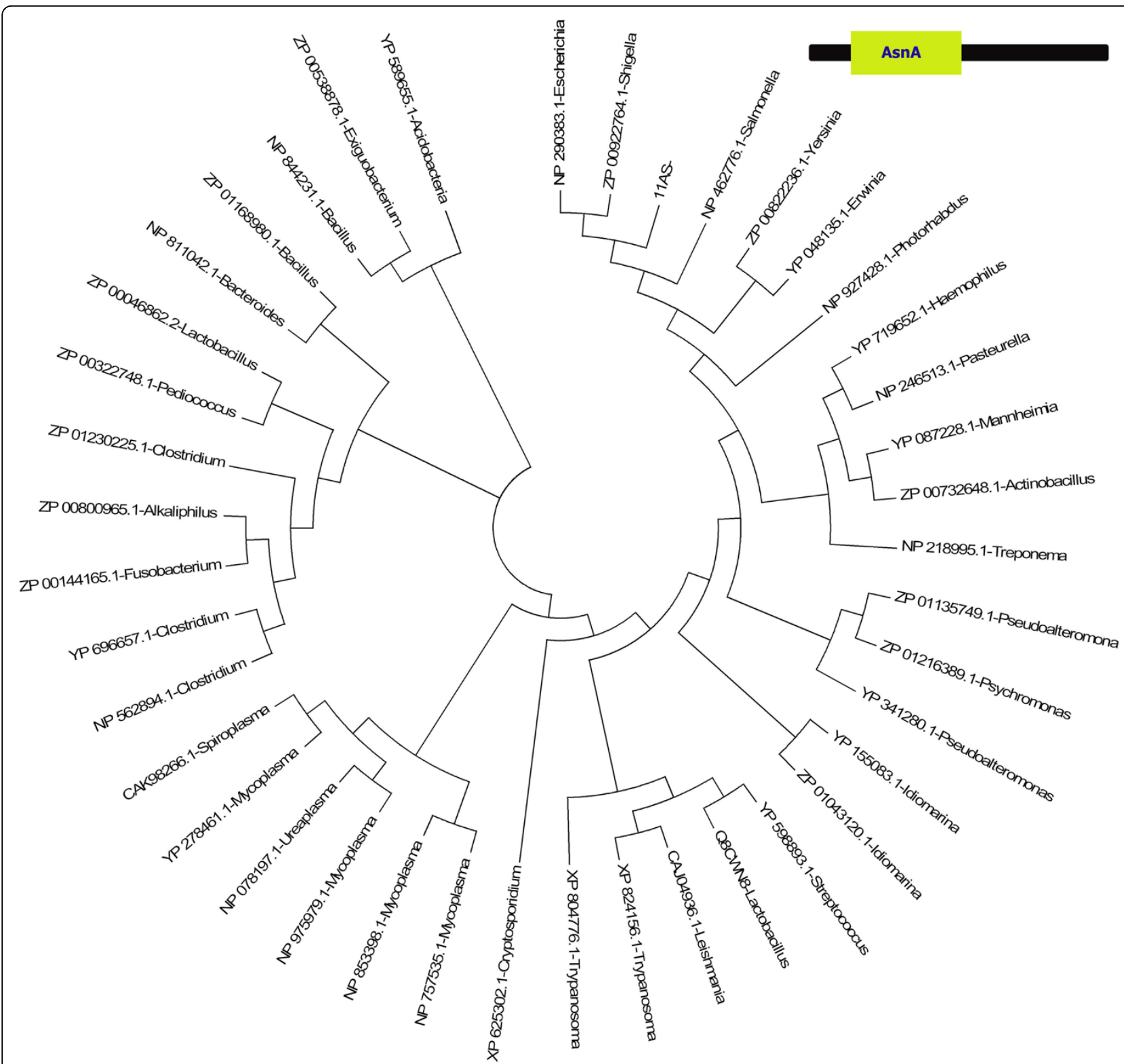

Figure 3 Phylogenetic tree of homologues obtained in the PSI-BLAST search. Domain architecture is shown on the top-right. All the homologues have identical domain architecture with amino-terminal AsnA domain. The mode of deriving phylogenetic trees is as described in Methods.

In another example, NP 853462.1 protein has an unassigned region (1-265) in the $\mathrm{N}$-terminal region followed by a C-terminal existing Peptidase_S8 domain. All the PSI-BLAST hits were from prokaryotes. The alignment (Figure 4) and conservation of secondary structures in this region suggests the existence of functionally unidentified domain that could augment the basic peptidase activity. It is also interesting to note that all the homologous sequences have similar domain architectures.
In another protein NP_852844.1, we had analysed an unassigned region from residues 38 to 109 and the gene product already was associated with KOW domain at the $\mathrm{N}$-terminus (from 5 to 37 residues). 109 homologues could be identified by PSI-BLAST and all homologues belong to prokaryotic organisms (Figure 5). All the homologous sequences have similar predicted secondary structure content. Most of the homologues also have similar domain architecture with $\mathrm{N}$-terminal $\mathrm{KOW}$ motif and $\mathrm{C}$ - terminus as an unassigned region. KOW 


\section{Consensus}

ZP_00229693.1-Listeria ZP_00799834.1-Alkaliphilus ZP_00062925.2-Leuconostoc NP_976020.1-Mycoplasma *NP_853462.1.1-265-Mycoplasma Consensus

ZP_00229693.1-Listeria ZP_00799834.1-Alkaliphilus ZP_00062925.2-Leuconostoc NP_976020.1-Mycoplasma *NP_853462.1.1-265-Mycoplasma Consensus

ZP_00229693.1-Listeria ZP_00799834.1-Alkaliphilus ZP 00062925.2-Leuconostoc NP_976020.1-Mycoplasma *NP_853462.1.1-265-Mycoplasma Consensus

ZP_00229693.1-Listeria ZP_00799834.1-Alkaliphilus ZP_00062925.2-Leuconostoc NP_976020.1-Mycoplasma *NP_853462.1.1-265-Mycoplasma Consensus

ZP_00229693.1-Listeria ZP_00799834.1-Alkaliphilus ZP_00062925.2-Leuconostoc NP_976020.1-Mycoplasma *NP_853462.1.1-265-Mycoplasma Consensus

ZP_00229693.1-Listeria ZP_00799834.1-Alkaliphilus ZP_00062925.2-Leuconostoc NP_976020.1-Mycoplasma *NP_853462.1.1-265-Mycoplasma P S I GL

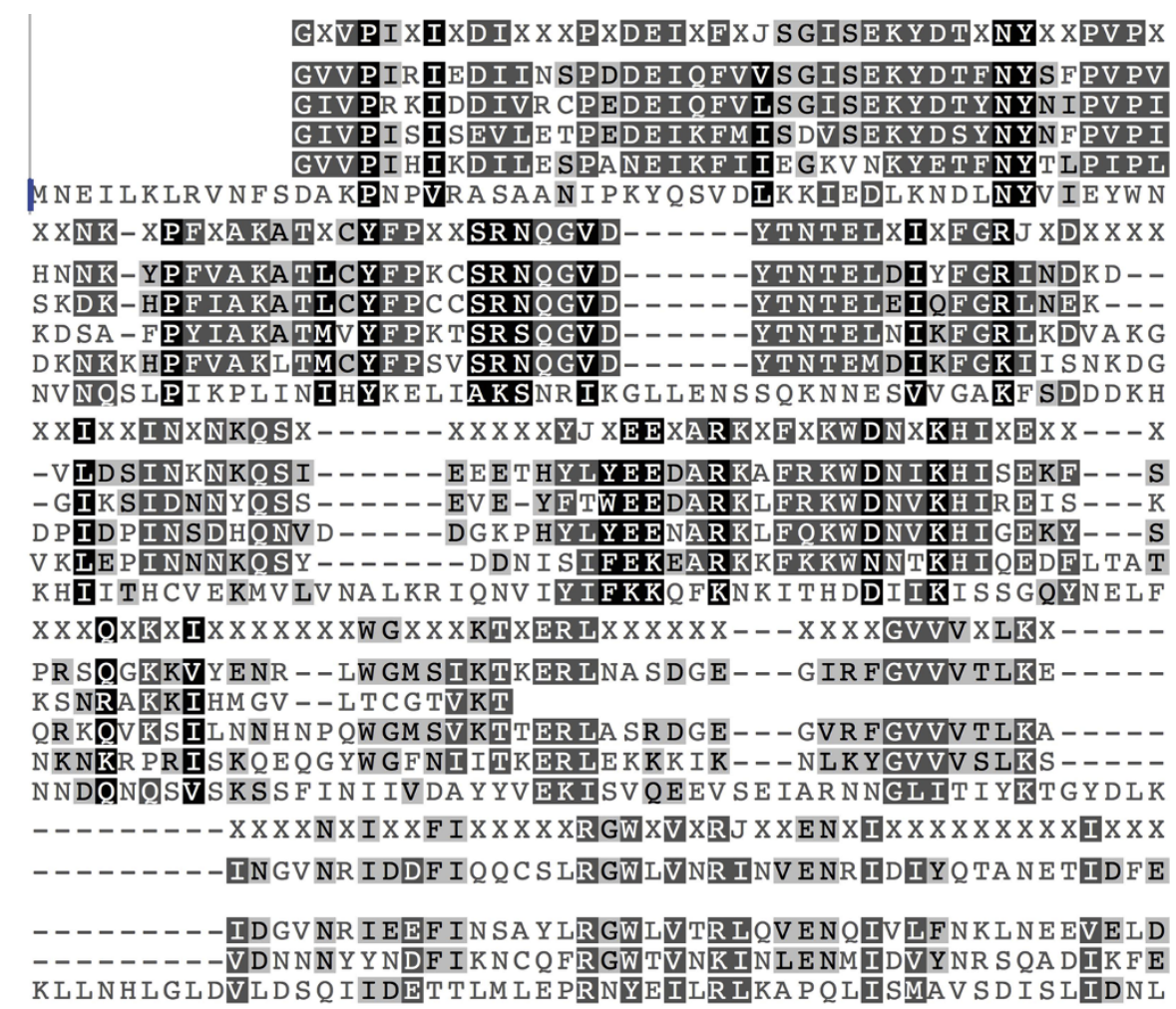

GXVPIXIXDIXXXPXDEIXFxJ SGISEKYDTXNYXXPVPx GVVPIRTEDIINSPDDEIOFVVSGISEKYDTFNYSFPVPV GIVPR KIDDIVR CPEDEIOFVLS GISEKY DTYNYN IPVP I G IVPISISEV LE TPEDEI KFMISDVSEKYDSYNYNF PVP I GVVPIHIKD I LESPANE I KF I IEGKVNKYETFNYTLP I PL M NE I L K LRV NF S DA KPN PVRA SAA N I P KY QSV DLK KIEDL KN D LNYV IEYWN $x \times \mathbb{N K}-X$ PF XAKATX CYF P X XSRNOGVD--- - -YTNTELXIXF GR J XDXXX -YPFVA KATICYF PKCSRNOGVD---- -YTNTELDTYF GR INDKD - S KDK-HPF IAKATLCYF PC CSR NOGVD----- YTNTELETQF GRLNEK--DKNKK HPFVAKLTMCYF PSVSR NOGVD-..-- -YTNTEMDIKFGKII SNKDG

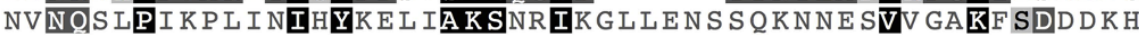

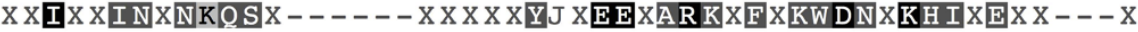
-VIDSINKNKOSI - - - - - EE E THY LYEEDARKAFR KWDN IKH ISEKF- - S V KIEP I NNNKOSY-- - - - D DN I SIF EKEAR KKF K KWNNT KH IOEDFLTA T K HII TH CV E KMV LV NA L KR I QNV IY IF K KO F KN K I T H DDIIKI S S GQYN E L F

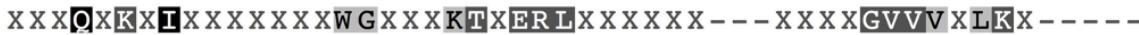
PR SDGKKVYENR - - LW GM SIKTKER LNA S D GE - - - GIR FGVVV T LKE - - - - S NKNKR PRIS KOEOGYW GFNIITKER LEKKKIK- - - NLKYGVVV S IKS - - - N NDQNOSVISKS S F I N I IV DA Y YVEK ISV QEEV S E IAR NNGL IT I YKT G Y D L K

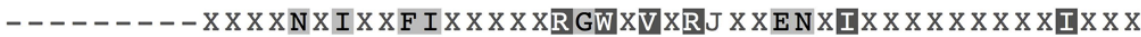
- - - - - - - VDNNNYYNDF I KNCOFR GWTVNK INLENMIDVYNR SOA DIKFE

Figure 4 Multiple sequence alignment of unassigned region NP_853462.1.1-265 indicated by ${ }^{* \prime}$ in the alignment and its homologues obtained in the PSI-BLAST search. Consensus sequence showed on the top the alignment and the species names given along with sequence ids.

motif is only about 35 residues long and links a bacterial transcription factor with ribosomal proteins[17]. The presence of conserved residues, with twice the size of KOW motif at the C-terminal region, suggests the functional role of $\mathrm{C}$-domain in additionally stabilizing the oligomeric assemblies and thereby perhaps contributing to improved efficiency of protein expression.

The results presented in this paper, are based on Pfam21 database with 8957 protein domain families; updated version of Pfam database (Pfam23) has 10340 domain families. Comparison of 71 newly predicted domains in the 62 unassigned regions to the updated Pfam23 database revealed that 67 domains, out of 71 , still remain unassigned in the Pfam 23 database. Among four Pfam23 annotations, three are the same as our earlier prediction and one annotation differs from our prediction. Comparison of our results with conserved domain database (CDD v2.16)[18] revealed that 34 out of 71 unassigned regions remained unassigned in the CDD database also, whereas remaining 37 regions are annotated as domains. Out of these 37 annotated domains in the CDD database, 25 domains are the same as predicted by our method described above. Assignments which differ from the new Pfam database (Pfam23) and CDD (seeming false positives and false negatives) were analyzed further. 12 out of 71 PURE predicted domains differ when compared to CDD database (Table 2); These differences could arise due to inherent differences in the methodologies of CDD and Pfam database construction. When compared with Pfam 23 database, out of 71 PURE predictions, 67 still remain unassigned and three agreed up on the PURE predictions. But, there is one disagreement: 124 residue-long protein NP_853458.1 was full-length unassigned sequence and PURE method assigned 'VapD_N' domain with E value of 0.006 . In the Pfam23 database, 'CRISPR_Cas2' domain is assigned to the sequence with $E$ value of $3.4 \mathrm{e}^{-47}$. This 


\begin{tabular}{|c|c|}
\hline \multicolumn{2}{|l|}{ Consensus } \\
\hline 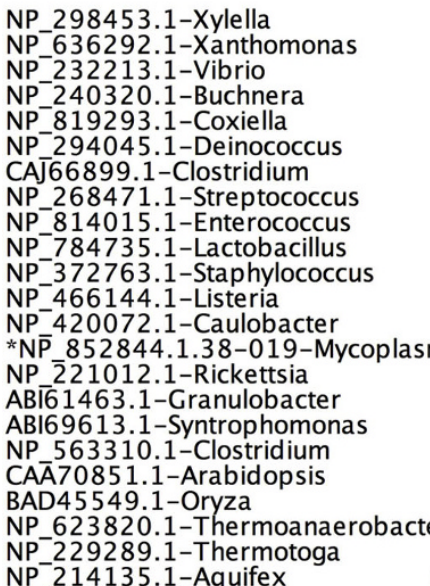 & 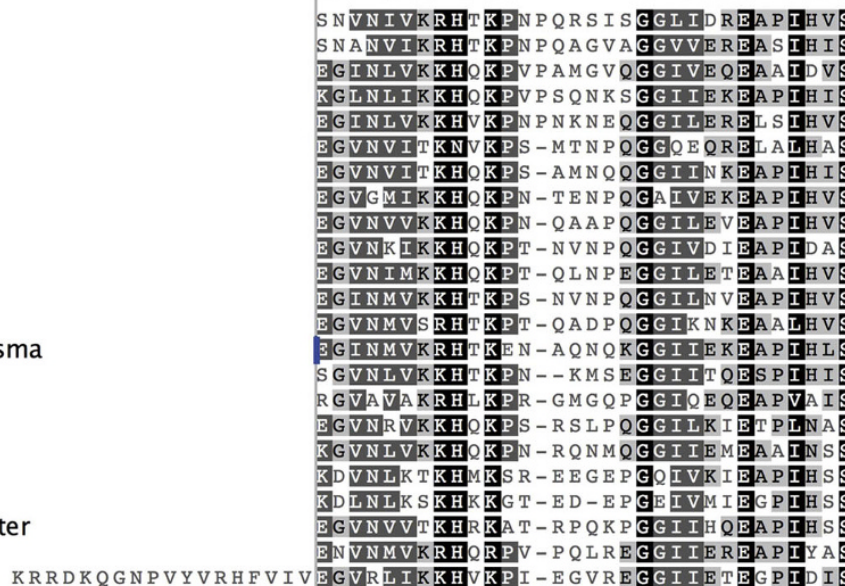 \\
\hline & 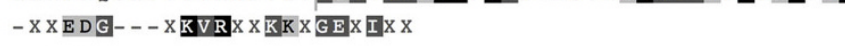 \\
\hline 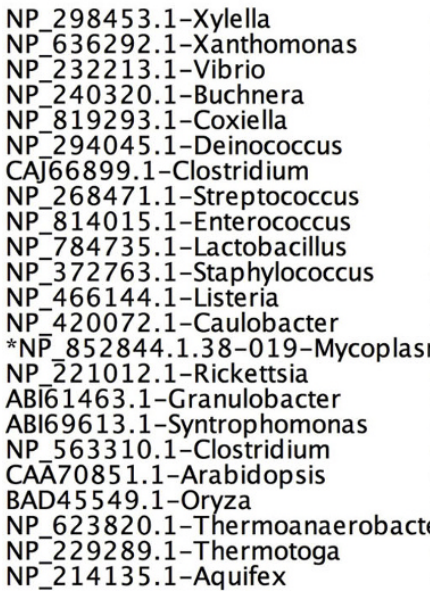 & 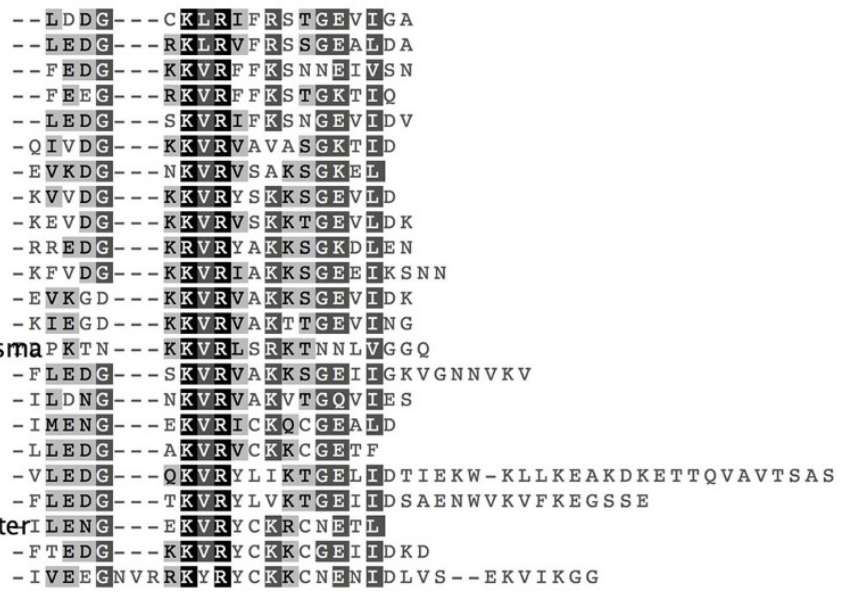 \\
\hline
\end{tabular}

Figure 5 Multiple sequence alignment of unassigned region NP_852844.1.39-109, (indicated by '*' in the alignment) and its homologues obtained in the PSI-BLAST search. Consensus sequence is shown on the top of the alignment.

Table 2 Comparison of PURE predicted domains with CDD predicted domains

\begin{tabular}{llll}
\hline S.No & Seq id & PURE & CDD \\
\hline 1 & NP_852935.1 & antocodon_1 - 55-218 & MetG - 10-199 \\
\hline 2 & NP_853333.1 & LMP - 1260-1320 & SbcC - 1253-1856 \\
\hline 3 & LMP - 1420-1580 & \\
\hline 4 & LMP - 1600-1760 & Type I site-specific restriction-modification system- 2-1018 \\
\hline 5 & NP_853467.1 & Helicase_C - 660-730 & mRNA degradation ribonucleases-23-594 \\
\hline 6 & NP_852865.1 & Lactamase_B - 40-248 & \\
\hline 7 & NP_852970.1 & MatE - 364-530 & NorM - 127-558 \\
\hline 8 & NP_853282.1 & MFS_1 - 480-992 & SecD - 359-574 \\
\hline 9 & NP_853404.1 & THUMP - 78-170 & PseudoU_synth - 112-167 \\
\hline
\end{tabular}

Sequence id in the second column, PURE predicted domains in the third column, domain name followed by starting and ending residue of the domain shown. In the last column CDD predicted domains name of the domain followed by domain range shown. 
could be due to the short length of 'VapD_N' (40 residues long) domain alignment and the borderline $\mathrm{E}$ value (0.006 and a cut-off 0.01). Moreover, the 'CRISPR_Cas2' (PF09827) domain family is defined only in Pfam23, but not in Pfam21 database.

The nature of sequence/domain searches is such that the databases are constantly going through updates and it is inevitable that our new findings might appear obsolete due to the constant updates of robust databases such as Pfam and CDD. Where there is concurrence with the newer version of a database, they serve to validate the approach. Where there is still new information obtained from PURE approach, this clearly suggests the value and novelty of the protocol due to the early realization of additional domains. When the newer entries are substantially high, this is very encouraging for the development of the approach suggesting that this has promise for discovering hitherto unidentified domains.

\section{Conclusions}

Here, we present the results of the application of a new method for domain identification to full genome of an avian pathogen Mycoplasma gallisepticum. In spite of filters, such as evolutionary conservation and high predicted structural content, about $20 \%$ of orphan proteins contained in this genome could be annotated with a known functional domain using our approach. Interestingly, our analysis revealed several meaningful alignments, which could relate to as yet functionally unidentified set of domains. This could be very useful as a starting subset for further functional screening in wet lab experiments. Several improvements of the methodology will be addressed in future. Furthermore, cross-genome comparisons of the results from our procedure between Mycoplasma gallisepticum and other Mycoplasma species are currently being investigated.

\section{Methodology}

Complete protein sequences of Mycoplasma gallisepticum (Strain R) were obtained from National centre for Biotechnology Information website[19].

\section{Extraction of Unassigned regions}

Mycoplasma gallisepticum protein sequences were scanned against a dataset of Hidden Markov Models (HMMs) obtained from the PfamA database (Pfam version 21.0)[5] which consists of 8957 families, employing the HMMpfam of the HMMER suite[11], with E-value threshold set to 0.1 . Sequences or sequence regions, which are not associated with any domain in the above search, were considered as unassigned regions.

\section{Filtering of Unassigned regions}

The unassigned sequences thus obtained were subjected to different filtering steps.

a. To avoid false positives in the PSI-BLAST [20] search, we considered only unassigned sequences with at least 70 residues long.

b. Transmembrane regions were excluded by using HMMTOP [21] and coiled-coil regions by using COILS[22] from the unassigned sequences. The above steps carried out to avoid non-specific hits in the PSI-BLAST [20] search.

c. Standalone version of protein secondary-structure prediction program PSIPRED[23] was used to predict the $\alpha$-helical, $\beta$-strand and coil (loop) content of different amino acids of unassigned regions. We employ $15 \%$ predicted secondary structural content as the minimum value, consistent with earlier work [13].

\section{PSI-BLAST searches}

The unassigned sequences, which have fulfilled the filtering criteria, were used to query non-redundant sequence database [24] employing PSI-BLAST [20], with low complexity filter turned on (E value cut off 0.001 ), to obtain homologues.

\section{HMMpfam search}

Only regions of homologues that aligned well with the query sequence were obtained from PSI-BLAST output to scan against a dataset of Hidden Markov Models (HMMs), which were obtained from the PfamA database (Pfam version 21.0)[5] which consists of 8957 families, employing HMMpfam of HMMER suite [11].

The indirect association of query sequence through homologous sequences with HMMs in the Pfam database gave rise to the definitions of full and partially associated domains. At least $75 \%$ of HMM should indirectly align with query to be considered as a fully associated domain and rest were considered as partially associated domains.

\section{Multiple sequence alignments}

Multiple sequence alignments of the query unassigned region and seed sequences of predicted domains which were obtained from Pfam[9] were performed using CLUSTALW program [14]. Multiple sequence alignments of the query unassigned region and hits in the PSI-BLAST were also performed when unassigned regions, where hits were obtained by PSI-BLAST search, but not in HMMpfam search. When necessary, alignments were optimized by manual editing. Phylogenetic trees were calculated using Neighbor-Joining (NJ) method [25]. Phylogenetic tree was plotted using MEGA package [26]. 
Additional file 1: Supplemental Table ST1 Predicted putative new domain families. In our analysis, some of the unassigned regions shared homologues (at least 5 hits in PSI-BLAST search), but failed to associate with any Pfam domain in Hmmpfam search. We performed multiple sequence alignments to assess sequence conservation. 63 unassigned regions showed good conservation which may indicate putative new domain family. In the table, second column indicates the protein-id and third column (UR = Unassigned Region) indicates the start and end points of unassigned regions. Fourth column indicates the percentage secondary structural content in the unassigned region by using PSIPRED program. Fifth column indicates the total number of hits in the PSIBLAST search and the sixth column indicates the number of PDB hits in PSI-BLAST search. Seventh column indicates the number of different species among the PSI-BLAST hits and eighth column indicates number of transmembrane helices which indicates whether protein is likely to be membrane-bound or not.

Additional file 2: Supplemental Table ST1 PfamB associations in the unassigned regions. 63 unassigned sequences were used to search against PfamB database (Pfam 24), only 20 unassigned sequences were associated with at least one PfamB domain. Unassigned sequence id along with start and end residues are showed in column two, PfamB start and end residue numbers are shown in column three, PfamB ids are shown in column four and the Expectation values with which PfamB domains associated are shown in the last column.

\section{Acknowledgements}

CSRC is supported by a PhD grant from Conseil Regional de La Reunion. This work is in part supported by PPF FRROI (Bioinflam) from University of La Reunion and French Ministry of Research. BO is thankful to Conseil Regional de La Reunion for financial support. RS thanks University de La ReUnion for the visiting professorship position and NCBS for financial and infrastructural support.

\section{Author details}

'Université de La Réunion, Equipe de Bioinformatique, Laboratoire de Biochimie et Génétique Moléculaire, 15 ave René Cassin, 97715 Saint Denis Messag Cedex 09, La Réunion, France. ${ }^{2}$ National Centre for Biological Sciences, GKVK Campus, Bellary Road, 560065 Bangalore, India.

\section{Authors' contributions}

CSRC has developed algorithms and codes used to identify domains and performed analysis on the whole genome of Mycoplasma gallisepticum. SSR compared the results with other domain databases. BO helped to device the experiments and to interpret analysis. RS devised the whole strategy for domain identification using indirect approach and supervised the work of CSRC. CSRC wrote the first draft of the manuscript. Both BO and RS contributed to the writing and critical reading of the manuscript. All authors read and approved the final manuscript.

\section{Competing interests}

The authors declare that they have no competing interests.

Received: 3 June 2009 Accepted: 12 April 2010 Published: 12 April 2010

\section{References}

1. Dietmann S, Holm L: Identification of homology in protein structure classification. Nat Struct Biol 2001, 8(11):953-957.

2. Orengo $C A$, Michie $A D$, Jones $S$, Jones DT, Swindells MB, Thornton JM: CATH-a hierarchic classification of protein domain structures. Structure 1997, 5(8):1093-1108

3. Murzin AG, Brenner SE, Hubbard T, Chothia C: SCOP: a structural classification of proteins database for the investigation of sequences and structures. J Mol Biol 1995, 247(4):536-540.

4. Sowdhamini R, Blundell TL: An automatic method involving cluster analysis of secondary structures for the identification of domains in proteins. Protein SCi 1995, 4(3):506-520.

5. Finn RD, Mistry J, Schuster-Bockler B, Griffiths-Jones S, Hollich V, Lassmann T, Moxon S, Marshall M, Khanna A, Durbin R, et al: Pfam: clans, web tools and services. Nucleic Acids Res 2006, , 34 Database: D247-251.
6. Reddy CC, Shameer K, Offmann BO, Sowdhamini R: PURE: a webserver for the prediction of domains in unassigned regions in proteins. $B M C$ Bioinformatics 2008, 9:281.

7. Reddy CC, Shameer K, Offmann BO, Sowdhamini R: PURE: A web server for querying the relationship between Pre-existing domains and Unassigned Regions in proteins. 2007 [http://www.natureprotocols.com/ 2007/11/01/pure_a_web_server_for_querying.php].

8. Papazisi L, Gorton TS, Kutish G, Markham PF, Browning GF, Nguyen DK, Swartzell S, Madan A, Mahairas G, Geary SJ: The complete genome sequence of the avian pathogen Mycoplasma gallisepticum strain $R$ (low). Microbiology 2003, 149(Pt 9):2307-2316.

9. Pfam Genome Distribution website. [http://www.sanger.ac.uk]

10. Reddy CS, Manonmani A, Babu M, Sowdhamini R: Enhanced structure prediction of gene products containing class III adenylyl cyclase domains. In Silico Biol 2006, 6(5):351-362.

11. Eddy SR: Profile hidden Markov models. Bioinformatics 1998, 14(9):755-763.

12. Sonnhammer EL, Eddy SR, Birney E, Bateman A, Durbin R: Pfam: multiple sequence alignments and HMM-profiles of protein domains. Nucleic Acids Res 1998, 26(1):320-322.

13. Rost B, Sander C, Schneider R: Redefining the goals of protein secondary structure prediction. J Mol Biol 1994, 235(1):13-26

14. Thompson JD, Higgins DG, Gibson TJ: CLUSTAL W: improving the sensitivity of progressive multiple sequence alignment through sequence weighting, position-specific gap penalties and weight matrix choice. Nucleic Acids Res 1994, 22(22):4673-4680.

15. Park J, Teichmann SA, Hubbard T, Chothia C: Intermediate sequences increase the detection of homology between sequences. J Mol Biol 1997, 273(1):349-354.

16. Nakatsu T, Kato H, Oda J: Crystal structure of asparagine synthetase reveals a close evolutionary relationship to class II aminoacyl-tRNA synthetase. Nat Struct Biol 1998, 5(1):15-19.

17. Kyrpides $N C$, Woese $C R$, Ouzounis CA: KOW: a novel motif linking a bacterial transcription factor with ribosomal proteins. Trends Biochem Sci 1996, 21(11):425-426.

18. Marchler-Bauer A, Panchenko AR, Shoemaker BA, Thiessen PA, Geer LY, Bryant SH: CDD: a database of conserved domain alignments with links to domain three-dimensional structure. Nucleic Acids Res 2002, 30(1):281-283.

19. National center for Biotechnology Information web site. [http://www ncbi.nlm.nih.gov/].

20. Altschul SF, Madden TL, Schaffer AA, Zhang J, Zhang Z, Miller W, Lipman DJ: Gapped BLAST and PSI-BLAST: a new generation of protein database search programs. Nucleic Acids Res 1997, 25(17):3389-3402.

21. Tusnady GE, Simon I: The HMMTOP transmembrane topology prediction server. Bioinformatics 2001, 17(9):849-850.

22. Lupas A, Van Dyke M, Stock J: Predicting coiled coils from protein sequences. Science 1991, 252(5010):1162-1164.

23. McGuffin LJ, Bryson K, Jones DT: The PSIPRED protein structure prediction server. Bioinformatics 2000, 16(4):404-405.

24. Wheeler DL, Barrett T, Benson DA, Bryant SH, Canese K, Chetvernin V, Church DM, DiCuccio M, Edgar R, Federhen S, et al: Database resources of the National Center for Biotechnology Information. Nucleic Acids Res 2006, (34 Database):D173-180.

25. Saitou N, Nei M: The neighbor-joining method: a new method for reconstructing phylogenetic trees. Mol Biol Evol 1987, 4(4):406-425.

26. Tamura K, Dudley J, Nei M, Kumar S: MEGA4: Molecular Evolutionary Genetics Analysis (MEGA) software version 4.0. Mol Biol Evol 2007, 24(8):1596-1599.

doi:10.1186/1756-0500-3-98

Cite this article as: Reddy et al:: Systematic search for putative new domain families in Mycoplasma gallisepticum genome. BMC Research Notes 2010 3:98. 